\title{
Characterization of the Mechanism of Action of RDR01752, a Novel Corrector of F508del-CFTR
}

\author{
Miquéias Lopes-Pacheco ${ }^{1}$, Iris Silva ${ }^{1}$, Mark Turner $^{2}$, Graeme Carlile ${ }^{2}$, Elvira Sondo ${ }^{3}$, David \\ Thomas $^{4}$, Nicoletta Pedemonte ${ }^{3}$, John Hanrahan ${ }^{2}$, and Margarida D. Amaral ${ }^{1}$ \\ ${ }^{1}$ Faculty of Sciences, Center for Biodiversity, Functional, and Integrative Genomics, \\ University of Lisboa, Campo Grande, 1749-016 \\ ${ }^{2}$ McGill University \\ ${ }^{3}$ Istituto Giannina Gaslini \\ ${ }^{4}$ McGill University Faculty of Medicine
}

April 28, 2020

\begin{abstract}
Background and Purpose: Despite progress in developing pharmacotherapies to rescue F508del-CFTR, the most prevalent Cystic Fibrosis (CF)-causing mutation, individuals homozygous for this mutation still face several disease-related symptoms. Thus, more potent compound combinations are still needed. Here, we investigated the mechanism of action (MoA) of RDR01752, a novel F508del-CFTR trafficking corrector. Experimental approach: F508del-CFTR correction by RDR01752 was assessed by biochemical, immunofluorescence microscopy and functional assays in cell lines and in intestinal organoids. To determine the MoA of RDR01752, we assessed its additive effects to those of genetic revertants of F508del-CFTR, the FDA-approved corrector drugs VX-809 and VX-661, and low temperature. Key Results: Our data demonstrated that RDR01752 rescues F508del-CFTR processing and plasma membrane (PM) expression to similar levels of VX-809 in cell lines, although RDR01752 produced lower functional rescue. However, in functional assays using intestinal organoids (F508del/F508del), RDR01752, VX-809 and VX-661 had similar efficacy. RDR01752 demonstrated additivity to revertants 4RK and G550E, but not to R1070W, as previously shown for VX-809. RDR01752 was also additive to low temperature. Co-treatment of RDR01752 and VX-809 further increased F508del-CFTR function compared to each corrector alone, but not PM expression. Conclusion and Implications: The lack of additivity of RDR01752 with the genetic revertant R1070W suggests that this compound has the same effect as the insertion of tryptophan at 1070, i.e., filling the pocket at the NBD1:ICL4 interface in F508del-CFTR, similarly to VX-809. Combination of RDR01752 with correctors mimicking the rescue by revertants G550E or 4RK could thus maximize rescue of F508del-CFTR.
\end{abstract}

\section{Characterization of the Mechanism of Action of RDR01752, a Novel Corrector of F508del- CFTR}

Running title: Rescue of F508del-CFTR by corrector RDR01752

Miquéias Lopes-Pacheco ${ }^{1}$, Iris A. L. Silva ${ }^{1}$, Mark J. Turner ${ }^{2,4}$, Graeme W. Carlile ${ }^{3,4}$, Elvira Sondo ${ }^{5}$, David Y. Thomas ${ }^{3,4}$, Nicoletta Pedemonte ${ }^{5}$, John W. Hanrahan ${ }^{2,4}$, Margarida D. Amaral ${ }^{1}$

${ }^{1}$ University of Lisboa, Faculty of Sciences, BioISI - Biosystems \& Integrative Sciences Institute, Lisboa, Portugal

${ }^{2}$ Department of Physiology, McGill University, Montréal, Québec Canada

3 Department of Biochemistry, McGill University, Montréal, Québec Canada

4 CF Translational Research Centre, McGill University, Montréal, Québec Canada 
${ }^{5}$ UOC, Genetica Medica, IRCCS Istituto Giannina Gaslini, Genova, Italy

\section{Counts:}

Abstract: 248

Main text: 4133

References: 40

Main Figures: 7

Supplementary Figures: 2

\section{Acknowledgements}

Work funded by UIDB/04046/2020 and UIDP/04046/2020 centre grants (to BioISI) from FCT, Portugal and research grant ERARE15-pp-010/JTC 2015 INSTINCT from FCT, Portugal (to MDA) and from CF Canada, FRQS and CIHR (to JWH and DYT). The authors thank Sofia Correia and Luís Marques (both from BioISI) for technical support and Cystic Fibrosis Foundation Therapeutics (CFFT, USA) for C18 compound and 596 anti-CFTR antibody.

\section{Conflict of interest statement}

There is no conflict of interest related to this work.

ABSTRACT (248 words; $\max 250$ )

Background and Purpose:Despite progress in developing pharmacotherapies to rescue F508del-CFTR, the most prevalent Cystic Fibrosis (CF)-causing mutation, individuals homozygous for this mutation still face several disease-related symptoms. Thus, more potent compound combinations are still needed. Here, we investigated the mechanism of action (MoA) of RDR01752, a novel F508del-CFTR trafficking corrector.

Experimental approach: F508del-CFTR correction by RDR01752 was assessed by biochemical, immunofluorescence microscopy and functional assays in cell lines and in intestinal organoids. To determine the MoA of RDR01752, we assessed its additive effects to those of genetic revertants of F508del-CFTR, the FDA-approved corrector drugs VX-809 and VX-661, and low temperature.

Key Results: Our data demonstrated that RDR01752 rescues F508del-CFTR processing and plasma membrane (PM) expression to similar levels of VX-809 in cell lines, although RDR01752 produced lower functional rescue. However, in functional assays using intestinal organoids (F508del/F508del), RDR01752, VX-809 and VX-661 had similar efficacy. RDR01752 demonstrated additivity to revertants 4RK and G550E, but not to R1070W, as previously shown for VX-809. RDR01752 was also additive to low temperature. Co-treatment of RDR01752 and VX-809 further increased F508del-CFTR function compared to each corrector alone, but not PM expression.

Conclusion and Implications: The lack of additivity of RDR01752 with the genetic revertant R1070W suggests that this compound has the same effect as the insertion of tryptophan at 1070, i.e., filling the pocket at the NBD1:ICL4 interface in F508del-CFTR, similarly to VX-809. Combination of RDR01752 with correctors mimicking the rescue by revertants G550E or 4RK could thus maximize rescue of F508del-CFTR.

Keywords: cystic fibrosis, protein trafficking, revertants, low temperature, intestinal organoids, drug discovery.

\section{INTRODUCTION}

Cystic fibrosis (CF) is the most common life-threating autosomal recessive disease among Caucasians, affecting almost 50,000 individuals in Europe (ECFS Patient Registry, 2017). It is caused by mutations in the gene encoding the CF transmembrane conductance regulator (CFTR) protein, which functions as a chloride $\left(\mathrm{Cl}^{-}\right)$and bicarbonate $\left(\mathrm{HCO}_{3}{ }^{-}\right)$channel at the apical plasma membrane (PM) of epithelial cells (Riordan, 
2008). CF-causing mutations cause channel dysfunction, leading to abnormal ion transport and dehydration of epithelia in several tissues (De Boeck \& Amaral, 2016; Lopes-Pacheco, 2016). Although CF is a multiorgan disease, the respiratory disorder represents the major cause of morbidity and mortality of individuals with CF due to airway obstruction by a thick mucus, chronic inflammation and persistent infections, which ultimately result in respiratory failure (De Boeck \& Amaral, 2016; Lopes-Pacheco, 2016).

CFTR protein is composed of two transmembrane domains (TMD1/2), two nucleotide-binding domains (NBD1/2) and a regulatory domain (RD). The TMDs form the pore through which anions are conducted along their electrochemical gradient, while the NBDs regulate channel gating by binding and hydrolyzing ATP and after RD phosphorylation at multiple sites. Interdomain interactions are critical for this complex protein to achieve its native conformation state (Riordan, 2008).

Over 2,000 CFTR gene variants have been reported so far (http://www.genet.sickkids.on.ca/), with deletion of a phenylalanine at position 508 (F508del in NBD1) being the most prevalent and occurring in $80 \%$ of individuals with CF in Europe, albeit with some geographic variability (ECFS Patient Registry, 2017). F508del causes CFTR protein misfolding that is recognized by the endoplasmic reticulum (ER) quality control (ERQC) machinery and prematurely degraded by the proteasome (Jensen et al., 1995). Rescue of F508del-CFTR was first demonstrated by low temperature incubation of cells heterologously expressing this mutant (Denninget al., 1992), thus proving that this mutant is both temperature sensitive and rescuable.

Over the past decade, significant efforts have been put into high-throughput screening (HTS) of small molecule libraries to identify compounds that rescue the F508del-CFTR protein to the PM. To date, there are three correctors approved for clinical use by the Food and Drug Administration (FDA), being two also approved by the European Medicine Agency (EMA) (all combined with potentiator VX-770/ivacaftor): VX809/lumacaftor, VX-661/tezacaftor and VX-445/elexacaftor (only FDA-approved). In clinical trials, individuals who were F508del-homozygous and treated with either VX-809 or VX-661 plus VX-770 demonstrated a significant, albeit modest, improvement in lung function (Wainwright et al., 2015; Taylor-Cousar et al. 2017). More recently, VX-445 was added to the co-treatment with VX-661/VX-770 and this triple combination demonstrated greater therapeutic benefit in phase 3 clinical trials (Heijerman et al., 2019; Middletonet al., 2019), thus leading to its FDA-approval in individuals with CF, aged [?]12 years and with the F508del mutation in at least one allele.

Despite such progress, individuals with CF still face several disease-related symptoms and complications, including a progressive deterioration of lung function, and thus novel correctors are still needed to achieve more potent combinations. Furthermore, there are other CFTR trafficking mutants that are not efficiently rescued by available correctors, including G85E and N1303K (Dekkers et al., 2016; Lopes-Pacheco et al., 2017). Along these lines, the novel RDR01752 compound was identified as a F508del-CFTR traffic corrector in a small-scale screen (Carlile et al., 2007) and demonstrated to thermally stabilize purified murine F508delNBD1 in vitro(Sampson et al., 2011). However, its mechanism of action (MoA) remains to be elucidated.

Here, we investigated the MoA of RDR01752 in cell lines stably expressing either F508del-CFTR or other CFTR mutants, and in F508del/F508del intestinal organoids. The MoA of RDR01752 was explored by analyzing its additive effects to those of previously described CFTR genetic revertants. These are secondsite mutations, i.e., in ciswith F508del that partially rescue F508del-CFTR. One of these revertants results from removal of the arginine-framed motifs (AFT) acting as retention signals (4RK), thus allowing the mutant protein to escape the ERQC (Chang et al., 1999; Roxo-Rosa et al., 2006; Farinhaet al., 2013). Two others work by correcting folding at critical structural pockets present in the 3D-structure of F508del-CFTR that are absent in wild-type (WT)-CFTR. These include G550E that acts by stabilizing the NBD1:NBD2 dimer interface (Roxo-Rosa et al.,2006) and R1070W that restores the NBD1:ICL4 interaction (Serohijoset al., 2008; Thibodeau et al., 2010; Farinha et al., 2013). We also investigated the effects of RDR01752 on the DD/AA variant on the background of WT-CFTR, which lacks the double diacidic code necessary for Sec24-CFTR association and ER exit and thus is retained in the ER but is not misfolded (Wang et al., 2004; Farinha et al., 2013). Finally, we tested RDR01752 in combination with low temperature and the FDA-approved corrector drugs VX-809/lumacaftor and VX-661/tezacaftor (and compound C18) with and 
without chronic exposure of the potentiator VX-770/ivacaftor.

\section{METHODS}

Cell culture. The CF bronchial epithelial (CFBE) cell line stably expressing F508del-CFTR was cultured as before (Canato et al.,2018). CFBE cells stably expressing mCherry-Flag-CFTR (WT, F508del, DD/AA variants or carrying G550E, R1070W, 4RK in cis with F508del) were cultured and CFTR expression was induced with doxycycline (Dox) $1 \mu \mathrm{g} / \mathrm{mL}$ as described (Botelho et al., 2015). Fischer rat thyroid (FRT) epithelial cells stably expressing CFTR variants (wt, G85E, R334W, T338I, R347P, F508del, V520F, S549F, G551D, M1101K, N1303K) were cultured as before (Turner et al., 2020). All cell lines were maintained in a humidified incubator at $5 \% \mathrm{CO}_{2}$ and $37^{\circ} \mathrm{C}$, except during low temperature experiments, in which cells were incubated at $27^{\circ} \mathrm{C}$ for $24 \mathrm{~h}$.

Chemicals . All reagents were of the highest purity available. Corrector compounds were either commercially obtained: RDR01752 (STK001879, Vitas-M Lab., IL, USA), VX-809, VX-661 and VX-770 (S1565, S7058 and S1144, Selleckchem, TX, USA) or from CFFT: C18. Correctors were diluted in dimethyl sulfoxide (DMSO) and added to cells diluted in 1\% FBS supplemented medium at indicated concentrations: 1 to

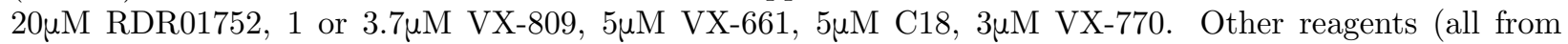
Sigma, MO, USA, in DMSO solutions) were (final concentrations, unless otherwise stated): $2 \mu \mathrm{M}$ forskolin

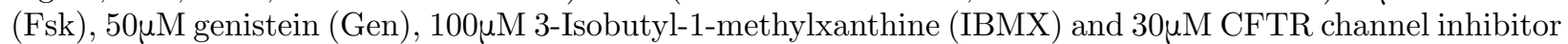
$\mathrm{CFTR}_{\text {Inh-172 }},\left(\operatorname{Inh}_{172}\right)$.

Western blotting (WB). Whole-cell lysates were subjected to SDS-PAGE 7\% (w/v) gel analysis followed by CFTR detection using monoclonal anti-human CFTR antibody (596 [1:3000] from CFFT) as previously (Farinha et al., 2013). Anti- $\alpha$-tubulin antibody (1:10,000, Sigma) was used as a loading control. CFTR quantification was as described (Canato et al., 2018) and its processing obtained by the ratio between the mature form and total CFTR (mature and immature forms).

Immunostaining. Twenty-four hours after seeding stably expressing mCherry-Flag-CFTR CFBE cells (carrying wt-, F508del-, DD/AA- or -G550E-F508del, R1070W-F508del, 4RK-F508del-CFTR) onto 384-well plates, compounds were concomitantly administered with Dox. After $48 \mathrm{~h}$, cells were immunostained and CFTR PM expression quantified as described (Botelho et al., 2015).

HS-YFP assay on the plate reader. Measurements of CFTR activity were carried out on CFBE cells expressing both F508del-CFTR and HS-YFP (YFP-H148Q/I152L) as described (Sondo et al., 2011). The assay consists of a continuous 14-s fluorescence read-out 2 s before and $12 \mathrm{~s}$ after injection of an iodidecontaining solution (PBS with $\mathrm{I}^{-}$at final concentration $100 \mathrm{mM}$ ). Fluorescence quenching rate of $\mathrm{I}^{-}$influx was determined for the final 11s of the data for each well and was fitted to an exponential function to extrapolate initial slope (Sondo et al., 2011).

FLIPR membrane potential (FMP) assay. Measurements of FMP to assess CFTR function were performed in FRT cells expressing various CFTR mutants as described (Carlile et al., 2018 ).

Micro-Ussing chamber measurements. Transepithelial electrical resistance (TEER) of CFBE cells growing on Snapwell inserts was measured as before (Awatade et al., 2015). Briefly, monolayers with resistance [?] $450 \Omega \mathrm{cm}^{2}$ were mounted in micro-Ussing chambers with perfusion for recordings under open-circuit conditions as described (Awatade et al.,2015). Changes in transepithelial voltage $\left(\mathrm{V}_{\text {te }}\right)$ were continuously recorded and equivalent Fsk/IBMX-stimulated short-circuit currents $\left(\mathrm{I}_{\text {eq-sc }}\right)$ were calculated by Ohm's law from $\mathrm{V}_{\text {te }}$ and $\mathrm{R}_{\mathrm{te}}\left(\mathrm{I}_{\mathrm{eq}-\mathrm{sc}}=\mathrm{V}_{\mathrm{te}} / \mathrm{R}_{\mathrm{te}}\right)$. For FRT cell lines, transepithelial voltage was measured at $37^{\circ} \mathrm{C}$ with continuous stirring by gassing with $95 \% \mathrm{O}_{2}$ and $5 \% \mathrm{CO}_{2}$ as described (Turner et al., 2020).

Organoid swelling assay. The forskolin-induced swelling (FIS) assay was performed as described (Dekkers et al., 2016). Twenty-four hours after seeding, organoids were stimulated with Fsk with or without a potentiator and live-cell imaging was performed using bright field microscopy (Leica DMI6000B) with a $5 \times$ objective for $60 \mathrm{~min}$ at $37^{\circ} \mathrm{C}$. For quantification of the area under the curve (AUC; $t=60 \mathrm{~min}$, baseline $=100 \%$ ) a 
CellProfiler-based algorithm was used (Hagemeijer et al., in preparation). Experiments were performed in triplicate and repeated 3-4 times.

Statistical analyses. Statistical comparisons were made using one-way ANOVA followed by Dunnett's or Tukey's post-hoc tests for parametric data or using Kruskal-Wallis test followed by Dunn's post-hoc test for nonparametric data (GraphPad Prism v.6.01). $P$ values $<0.05$ were considered significant.

\section{RESULTS}

\section{RDR01752 rescues F508del-CFTR processing, PM traffic and channel function}

Incubation of CFBE cells expressing F508del-CFTR with the RDR01752 compound rescued F508del-CFTR processing, resulting in the appearance of the fully-glycosylated form of CFTR ( $\sim 180 \mathrm{kDa}$, band C) in a dosedependent manner with the maximal correction achieved at $10 \mu \mathrm{M}$ (Fig. 1A,B). This effect was comparable to that obtained for VX-809 or VX-661, and in contrast to that obtained for DMSO (vehicle), which only led to the appearance of the core-glycosylated form of CFTR ( $\sim 140 \mathrm{kDa}$, band B). This result was also confirmed by the immunofluorescence detection of the Flag-tag of mCherry-Flag-F508del-CFTR expressed in CFBE cells without cell permeabilization, only in cells treated with RDR01752 or VX-809, but not DMSO (Fig. $1 \mathrm{C}, \mathrm{D})$.

To assess the ability of RDR01752 to restore F508del-CFTR function, we first measured the rate of HS-YFP quenching induced by iodide influx into cells in CFBE cells stably co-expressing F508del-CFTR and the HSYFP (Fig. 2A,B). Both RDR01752 (10 $\mu \mathrm{M})$ and VX-809 demonstrated rescue of F508del-CFTR function, although the efficacy of RDR01752 was lower than that of VX-809. In order to confirm these findings, we then investigated rescue of F508del-CFTR function by RDR01752 in polarized CFBE cells in the Ussing chamber (Fig. 2C-F). A significant increase in equivalent short-circuit current $\left(\Delta \mathrm{Isc}_{\mathrm{eq}}\right)$ was observed upon Fsk/IBMX stimulation in cells incubated with either RDR01752 or VX-809 and stimulated with potentiator genistein (Gen) versusthose incubated with DMSO alone. These data are consistent with the rate of HS-YFP quenching.

F508del/F508del intestinal organoids respond positively to RDR01752

Next, we tested the effects of RDR01752 using the FIS assay in intestinal organoids with the F508del/F508del genotype. Organoids were incubated with RDR01752, VX-809 or VX-661 for 24h and then acutely stimulated (30 min) with Fsk with or without a potentiator (VX-770 or Gen) to further enhance CFTR function (Figs. 3 and S1). Significant swelling was observed in organoids incubated with RDR01752, VX-809 or VX-661 and acutely stimulated with either VX-770 or Gen, in contrast to absence of swelling in organoids without any potentiator or DMSO control. Interestingly, similar swelling values were observed for organoids incubated with any corrector plus potentiator combination.

RDR01752 increases the rescue of F508del-CFTR PM in cells expressing in cis the genetic revertants G550E and $4 R K$ or low temperature, but not in $R 1070 \mathrm{~W}$ nor $D D / A A$

In order to characterize the mechanism of action (MoA) by which RDR01752 rescues F508del-CFTR, we investigated revertants of this mutant. To this end, CFBE cell lines stably expressing double tagged-F508delCFTR incis with the following genetic revertants: G550E, R1070W, or 4RK were incubated with this compound. In parallel CFBE cells expressing WT-CFTR or the traffic-null variant DD/AA (on a WT background) were also treated with RDR01752. Cells were incubated both at $37^{\circ} \mathrm{C}$ and low temperature $\left(27^{\circ} \mathrm{C}\right)$, and PM expression of each CFTR variant was assessed by immunofluorescence (Fig. 4A,B, respectively) and normalized to cells incubated with DMSO at $37^{\circ} \mathrm{C}$ (upper heatmap, first row).

Each of the correctors tested RDR01752, VX-809 or VX-661 rescued F508del-CFTR to the PM with similar efficacy (Fig. 4A) which was further enhanced when incubated at $27^{\circ} \mathrm{C}$ (Fig. 4B). These compounds also increased WT-CFTR PM expression, although VX-809 and VX-661 showed higher efficacy than RDR01752. A small additive effect was found for PM levels of wt-CFTR with each of these compounds and low temperature. 
Analysis of the effects on the revertants demonstrated that RDR01752, similarly to VX-809 and VX-661, is additive to G550E and 4RK in F508del-CFTR PM rescue (Fig.4A), and further additive to that of low temperature (Fig. 4B). In contrast, RDR01752, also similarly to VX-809 and VX-661, did not demonstrate additivity with R1070W (Fig. 4A), thus suggesting that, like VX-809 (Farinha et al., 2013), it might share a common mechanism. Furthermore, an increase in PM expression of DD/AA-CFTR variant was not observed (Fig. 4A), except when cells were incubated at low temperature (Fig. 4B), as before (Farinha et al., 2013), with no significant additive effect elicited by any of the correctors.

Rescuing of F508del-CFTR traffic by RDR01752 is not additive to VX-809 or VX-661

Next, we tested whether rescuing by RDR01752 is additive to that of other correctors, namely FDA-approved drugs VX-809 and VX-661 (Fig. 5) or C18 (Fig. S2). We also evaluated whether chronic exposure to VX-770 affects the rescue of F508del-CFTR by RDR01752 (Figs. 5 and S2). CFBE cells stably expressing mCherryFlag-F508del-CFTR were incubated with each corrector for $48 \mathrm{~h}$ and CFTR PM levels were quantified as above. None of the two-corrector combinations further enhance F508del-CFTR PM expression compared to each corrector alone (Fig. 5). Similarly to VX-809 or VX-661, rescue of F508del-CFTR by RDR01752 under chronic exposure to a relatively high concentration of VX-770 resulted in a decrease of F508del-CFTR PM expression (Fig. S2). The inhibitory effect of chronic exposure to VX-770 on F508del-CFTR rescue by VX-809 or VX-661 exposure observed here, as previously reported by others (Cholonet al., 2014; Veit et al., 2014), was even more pronounced for any combination of two correctors.

Functional rescue of other CFTR mutants by RDR01752

To evaluate the ability of RDR01752 to rescue other CFTR mutants, we used an FMP assay in FRT cells to measure the depolarization that occurs when CFTR PM channels are activated. The nine missense mutants studied are located across the different CFTR domains, namely in: TMD1 (G85E, R334W, T338I and R347P); NBD1 (V520F, S549F and G551D); TMD2 (M1101K); and NBD2 (N1303K). Cells expressing these CF-causing mutations were incubated with either RDR01752 or VX-809 for 24h (Fig. 6). An increase in the Fsk+Gen response was observed in F508del-expressing FRT cells treated with either RDR01752 or VX-809, indicating that CFTR function was rescued by RDR01752, consistent with both the $\Delta$ Isceq data $_{\text {en }}$ and the rate of HS-YFP quenching observed in CFBE cells. Both RDR010752 and VX-809 also rescue CFTR function in R334W-, V520F- and M1101K-expressing cells. However, in this assay only RDR01752 (and not VX-809) demonstrated an effect on G85E- and T338I-expressing cells, while R347P-, S549F- and N1303K-expressing cells only responded to VX-809 treatment, albeit the latter at very low levels (Fig. 6). No significant differences were observed in CFTR function between G551D- and WT-expressing cells after treatment with either RDR01752 or VX-809 compared to control.

To confirm these findings, we investigated the effects of RDR01752 and VX-809, alone or combined, in polarized FRT cells expressing the mutants that showed rescue, namely F508del-, G85E-, N1303K- or R334WCFTR by Ussing chamber measurements (Fig. 7). An increase of CFTR-dependent $\mathrm{Cl}^{-}$secretion was observed in F508del-expressing cells incubated with either VX-809 or RDR01752, albeit much lower for the latter. Incubation with both compounds combined resulted in a significantly greater rescue of F508del-CFTR function in comparison to VX-809 alone. Furthermore, although the rescue of R334W-CFTR by RDR01752 was not higher than that by VX-809 (as in the FMP assay), co-administering the two compounds did increase CFTR-dependent $\mathrm{Cl}^{-}$secretion. No significant effects were found in G85E- and N1303K-expressing cells in CFTR function after incubation with either RDR01752 or VX-809, alone or combined.

\section{DISCUSSION AND CONCLUSIONS}

The aim of this study was to characterize the effects of corrector RDR01752 both in cell lines that stably express F508del-CFTR or other rare CFTR mutations and in intestinal organoids that are F508del/F508del. Furthermore, we investigated the MoA of RDR01752 by evaluating its additivity with available corrector drugs, genetic revertants of F508del-CFTR, and low temperature.

Most CF drug development programs for CFTR modulators have focused on the rescue of F508del-CFTR 
as the most prevalent disease-causing mutant. Despite significant progress in restoring F508del-CFTR trafficking by corrector drugs, treatment of F508del-homozygous individuals with single correctors (VX-809 or VX-661) in combination with the potentiator VX-770 achieved only modest clinical improvements (Wainwright et al.,2015; Taylor-Cousar et al. 2017). Significantly greater therapeutic response was recently achieved by adding a second corrector (VX-445) to the previous combination (VX-661/VX-770) (Heijerman et al., 2019; Middleton et al., 2019), indicating that combination of correctors acting by distinct mechanisms is needed for efficient correction of F508del-CFTR and for clinical benefit of individuals with CF carrying this mutation. Although the MoA of VX-445 is still unknown, its additivity to VX-661 on F508del-CFTR rescue suggests that these compounds act by different modes.

Here, we looked into the MoA of the recently described F508del-CFTR corrector RDR01752 (Carlile et al., 2007). Our biochemical and immunofluorescence data demonstrated that RDR01752 rescues F508del-CFTR processing and PM expression in CFBE cells to levels similar to those of VX-809. However, in previous studies using baby hamster kidney (BHK) cells stably expressing F508del-CFTR, RDR01752 appeared to be less efficacious than VX-809 (Carlile et al., 2007, Sampson et al., 2011). Our functional data here, in both CFBE and FRT cell lines, also show that RDR01752 is less effective than VX-809 in restoring F508delCFTR-mediated $\mathrm{Cl}^{-}$secretion, consistent with previous findings in human bronchial epithelial (HBE) cells (F508del/F508del) (Carlile et al., 2018). Notwithstanding, as the validation of results in patient-derived specimens is an important step to provide a better prediction of the in vivo efficacy (Amaral and De Boeck, 2019; Lopes-Pacheco, 2020), we tested here this compound in intestinal organoids (F508del/F508del). Our data revealed that RDR01752 and VX-809/VX-661 can rescue F508del-CFTR function to similar levels in this assay. The higher efficacy observed in the organoids may derive from the fact that different cell systems were used and CFTR processing and function are influenced by the cell background and polarization state (Pedemonte et al., 2010; Rowe et al.,2010; Farinha et al., 2015).

To investigate the MoA of RDR01752 we analyzed its additivity to revertants that rescue F508del-CFTR by different mechanisms. RDR01752 effects were additive to G550E and 4RK, but not to R1070W as determined by rescue of F508del-CFTR protein to the PM. G550E and R1070W were proposed to act at two distinct CFTR interdomain contact points that are disrupted by F508del (Farinha et al., 2013): while G550E likely restores the NBD1:NBD2 dimerization interface (Roxo-Rosa et al.,2006), R1070W restores the NBD1:ICL4 interaction (Serohijos et al., 2008; Thibodeau et al., 2010). Thus, RDR01752, like VX-809, might act similarly to R1070W, i.e., by restoring the anchoring of ICL4 to the NBD1 surface by filling a pocket generated by the absence of F508, as the lack of additivity of these two compounds indicates. Indeed, the replacement of an arginine with a tryptophan at position 1070 (R1070W) helps restore interactions among the aromatic residue that were impaired by the lack of F508del at the NBD1 surface (Serohijoset al., 2008; Thibodeau et al., 2010; Farinha et al., 2013). RDR01752, like VX-809/VX-661, has a fused aromatic ring in its structure that can fit into this pocket left empty by F508del (Heet al., 2013; Farinha et al., 2013). This terminal aromatic ring in RDR01752 was also found to be critical for the stabilization of isolated F508del-NBD1 with an additional stabilization effect in presence of ATP, thus suggesting that RDR01752 does not bind to the ATP binding site in NBD1 (Sampson et al., 2011). This is also in agreement with the observed additivity of RDR01752 to G550E.

On the other hand, RDR01752 was also additive to 4RK. This variant (where four arginines in the AFT are simultaneously replaced to lysines) enables some F508del-CFTR protein to traffic to the PM by escaping the ERQC (Chang et al., 1999; Roxo-Rosa et al., 2006). Notably, although RDR01752, VX-809 and VX-661 rescued F508del-4RK-CFTR PM expression, their efficacy was distinct, with the additivity of RDR01752 to 4RK being higher than that of VX-809/VX-661. In turn, the latter correctors were more effective than RDR01752 in rescuing G550E-F508del-CFTR. Altogether, these data suggest that combinations of RDR01752 with compounds that mimic the correction induced by the G550E and 4RK revertants could maximize the rescue of F508del-CFTR.

Regarding the additivity to low temperature, RDR01752, VX-809 and VX-661 were similarly additive to $27^{\circ} \mathrm{C}$ incubation of F508del-expressing cells, consistent with previous reports demonstrating that VX-809 
was unable to restore the thermostability of F508del-CFTR (He et al., 2013). The three correctors, which were additive to $\mathrm{G} 550 \mathrm{E}$ and $4 \mathrm{RK}$, had an even greater effect when combined to low temperature. Although none of the correctors was additive to $\mathrm{R} 1070 \mathrm{~W}$ at $37^{\circ} \mathrm{C}$, additivity was observed for all three in combination to low temperature for this revertant, which was however, less pronounced for RDR01752. Altogether, these data indicate that despite the double correction effect of revertants and compounds on F508del-CFTR, there is still scope for further enhancement as indicated by low temperature data.

As with VX-809 and VX-661, RDR01752 was unable to overcome a Sec24-COPII-ER export defect of the diacidic variant DD/AA (on WT-CFTR backbone). Low temperature nevertheless enables DD/AA-CFTR to exit the ER through the conventional ER-to-Golgi pathway, since the rescued DD/AA-CFTR was found to be fully-glycosylated (Farinha et al.,2013). These data are compatible with the proposed mechanism for F508del-CFTR by low temperature (Farinha et al., 2013).

Although RDR01752 did not further enhance F508del-CFTR PM expression rescue by VX-809 or VX-661 in the immunofluorescence assay, it further increased the rescue of F508del-CFTR function when combined with VX-809versus each compound alone, which may be attributable to a weak potentiator activity of RDR01752 on F508del-CFTR channels (Sampsonet al., 2011). Notably, chronic VX-770 exposure reduced RDR01752rescued CFTR PM expression in F508del-expressing cells, as described for VX-809 and VX-661 (Cholon et al., 2014; Veitet al., 2014). However, chronic exposure of F508del-expressing cells to a low free concentration of VX-770 prevents the negative effect on VX-809-rescued CFTR (Matthes et al., 2016). Surprisingly, rescuing of CFTR PM expression by C18, a compound with a similar chemical structure to VX-809/VX-661 (Eckford et al., 2014; Lopes-Pacheco et al., 2016), was less affected by chronic VX-770 exposure. In fact, C18 and VX-809 possibly have a different MoA since in primary HBE cells, the former failed to rescue the trafficking mutant A561E-CFTR, in contrast to VX-809 (Awatade et al., 2014).

Because there are several CFTR mutations with the same trafficking defect as F508del-CFTR, i.e., class II (De Boeck \& Amaral, 2016, Lopes-Pacheco, 2020), but do not equally respond to the same CFTR corrector (Grove et al., 2011; Rapino et al., 2015, Dekkers et al., 2016; Lopes-Pacheco et al., 2017), we investigated whether RDR01752 could rescue some of these mutants. Functional assessment was initially performed by the FMP assay and then confirmed in Ussing chamber measurements of CFTR activity in polarized FRT cells stably expressing some of those CFTR mutations. As RDR01752 was demonstrated to be a weak potentiator of F508del-CFTR channels (Sampson et al., 2011), we tested its effect on R334W-CFTR, which has minimal trafficking impairment but reduced channel conductance that still allows for residual function (Sheppard et al., 1993). A slight but non-statistically significant increase in R334W-CFTR function was induced by RDR01752 whereas a greater effect was observed when combined with VX-809. We also tested RDR01752 on G85E and N1303K class II CFTR mutants which are temperature-insensitive despite having trafficking defects like F508del (Rapino et al., 2015; Lopes-Pacheco et al., 2017). N1303K was not rescued efficiently by RDR01752 or VX-809 when tested individually or in combination, consistent with previous findings in HBE cells (Awatade et al.,2014) and intestinal organoids (Dekkers et al., 2016). Regarding G85E, although it appeared to respond functionally to RDR01752 in the FMP assay, this was not confirmed in the Ussing chamber measurements. The lack of response of this mutant to several correctors has been previously reported (Grove et al., 2011; Lopes-Pacheco et al. 2017). Altogether, these data demonstrate that both G85E and N1303K trafficking defects are difficult to rescue and alternative correctors remain an unmet need for these mutants.

In conclusion, these data show that RDR01752, like VX-809/VX-661, rescues F508del-CFTR, albeit at lower efficiency, and like those two approved corrector drugs it does not rescue the G85E and N1303K traffic mutants. Our studies with revertants, aimed at understanding the MoA of this novel corrector, help explain such similarity in pharmaco-therapeutic behavior. Indeed, the data suggest RDR01752 may share a binding site on F508del-CFTR with VX-809 and VX-661, i.e., at the NBD1:ICL4 interface. The fact that RDR01752, like VX-809/VX-661, is additive to correction by the revertants G550E and 4RK and also by low temperature indicate that there is still scope for correctors to further increase the rescue of F508del-CFTR.

\section{REFERENCES}


Amaral MD, De Boeck K. (2019). Theranostics by Testing CFTR Modulators in Patient-Derived Materials: The Current Status and a Proposal for Subjects with Rare CFTR Mutations. J Cyst Fibros 18, 685-692.

Awatade NT, Uliyakina I, Farinha CM, Clarke LA, Mendes K, Solé A, et al. (2014). Measurements of Functional Responses in Human Primary Lung Cells as a Basis for Personalized Therapy for Cystic Fibrosis. EBioMedicine 2, 147-153.

Botelho HM, Uliyakina I, Awatade NT, Proença MC, Tischer C, Sirianant L, et al. (2015). Protein Traffic Disorders: An Effective High-Throughput Fluorescence Microscopy Pipeline for Drug Discovery. Sci Rep 5, 9038 .

Canato S, Santos JD, Carvalho AS, Aloria K, Amaral MD, Rune M, et al. (2018). Proteomic Interaction Profiling Reveals KIFC1 as a Factor Involved in Early Targeting of F508del-CFTR to Degradation. Cell Mol Life Sci 75, 4495-4509.

Carlile GW, Robert R, Zhang D, Teske KA, Luo Y, Hanrahan JW, et al. (2007). Correctors of protein trafficking defects identified by a novel high-throughput screening assay. Chembiochem 8, 1012-1020.

Carlile GW, Yang Q, Matthes E, Liao J, Radinovic S, Miyamoto C, et al. (2018). A Novel Triple Combination of Pharmacological Chaperones Improves F508del-CFTR Correction. Sci Rep 8, 11404.

Chang XB, Cui L, Hou YX, Jensen TJ, Aleksandrov AA, Mengos A, et al. (1999). Removal of multiple arginine-framed trafficking signals overcomes misprocessing of delta F508 CFTR present in most patients with cystic fibrosis. Mol Cell 4, 137-142.

Cholon DM, Quinney NL, Fulcher ML, Ester Jr CR, Das J, Dokholyan NV,et al. (2014). Potentiator of Ivacaftor Abrogates Pharmacologial Correction of $\triangle$ F508 CFTR in Cystic Fibrosis. Sci Transl Med 6, $246 \mathrm{ra} 96$.

De Boeck K, Amaral MD. (2016). Progress in Therapies for Cystic Fibrosis. Lancer Respir Med 4, 662-674.

Dekkers JF, Gogorza Gondra RA, Kruisselbrink E, Vonk AM, Janssens HM, de Winter-de Groot KM, et al. (2016). Optimal correction of distinct CFTR folding mutants in rectal cystic fibrosis organoids. Eur Respir $\mathrm{J} 48,451-458$.

Denning GM, Anderson MP, Amara JF, Marshall J, Smith AE, Welsh MJ. (1992). Processing of Mutant Cystic Fibrosis Transmembrane Conductance Regulator Is Temperature-Sensitive. Nature 358, 761-764.

Eckford PDW, Ramjeesingh M, Molinski S, Pasyk S, Dekkers JF, Li C,et al. (2014). VX-809 and Related Corrector Compounds Exhibit Secondary Activity Stabilizing Active F508del-CFTR After Its Partial Rescue to the Cell Surface. Cell Biol 21, 666-678.

ECFS Patient Registry - Annual Data Report 2017. Available at: https://www.ecfs.eu/sites/default/files/generalcontent-images/working-groups/ecfs-patient-registry/ECFSPR_Report2017_v1.3.pdf

Farinha CM, King-Underwood J, Sousa M, Correia AR, Henriques BJ, Roxo-Rosa M, et al. (2013). Revertants, Low Temperature, and Correctors Reveal the Mechanism of F508del-CFTR Rescue by VX-809 and Suggest Multiple Agents for Full Correction. Chem Biol 20, 943-955.

Farinha CM, Sousa M, Canato S, Schmidt A, Uliyakina I, Amaral MD. (2015). Increased Efficacy of VX-809 in Different Cellular Systems Results From an Early Stabilization Effect of F508del-CFTR. Pharmacol Res Perspect 3, e00152.

Grove DE, Fan C-Y, Ren HY, Cyr DM. (2011). The Endoplasmic Reticulum-Associated Hsp40 DNAJB12 and Hsc70 Cooperate to Facilitate RMA1 E3-dependent Degradation of Nascent CFTRDeltaF508. Mol Biol Cell 33, 301-314.

He L, Kota P, Aleksandrov AA, Cui L, Jensen T, Dokholyan NV, et al. (2013). Correctors of $\Delta$ F508 CFTR Restore Global Conformation Maturation Without Thermally Stabilizing the Mutant Protein. FASEB J 27, 
536-545.

Heijerman HGM, McKone EF, Downey DG, Braeckel EV, Rowe SM, Tullis E, et al. (2019). Efficacy and Safety of the Elexacaftor Plus Tezacaftor Plus Ivacaftor Combination Regimen in People with Cystic Fibrosis Homozygous for the F508del Mutation: a Double-Blind, Randomised, Phase 3 Trial. Lancet 394, 1940-1948.

Jensen TJ, Loo MA, Pind S, Williams DB, Goldberg AL, Riordan JR. (1995). Multiple Proteolytic Systems, Including the Proteasome, Contribute to CFTR Processing. Cell 83, 129-135.

Matthes E, Goepp J, Carlile GW, Luo Y, Dejgaard K, Billet A, et al. (2016). Low free drug concentration prevents inhibition of F508del CFTR functional expression by the potentiator VX-770 (ivacaftor). Br J Pharmacol 173, 459-470.

Middleton PG, Mall MA, Drevínek P, Lands LC, McKone EF, Polineni D, et al. (2019). Elexacaftor-TezacaftorIvacaftor for Cystic Fibrosis with a Single Phe508del Allele. N Engl J Med 381, 1809-1819.

Lopes-Pacheco M. (2016). CFTR Modulators: Shedding Light on Precision Medicine for Cystic Fibrosis. Front Pharmacol 7, 275.

Lopes-Pacheco M. (2020). CFTR Modulators: The Changing Face of Cystic Fibrosis in the Era of Precision Medicine. Front Pharmacol 10, 1662.

Lopes-Pacheco M, Boinot C, Sabirzhanova, Rapino D, Cebotaru L. (2017). Combination of Corectors Rescues CFTR Transmembrane-Domain Mutants by Mitigating Their Interactions with Proteostasis. Cell Physiol Biochem 41, 2194-2210.

Lopes-Pacheco M, Sabirzhanova I, Rapino D, Morales MM, Guggino WB, Cebotaru L. (2016): Correctors Rescue CFTR Mutations in Nucleotide-Binding Domain 1 (NBD1) by Modulating Proteostasis. Chembiochem 17, 493-505.

Pedemonte N, Tomati V, Sondo E, Galietta LJV. (2010). Influence of Cell Background on Pharmacological Rescue of Mutant CFTR. Am J Physiol Cell Physiol 294, C866-C874.

Rapino D, Sabirzhanova I, Lopes-Pacheco M, Grover R, Guggino WB, Cebotaru L. (2015). Rescue of NBD2 Mutants N1303K and S1235R of CFTR by Small-Molecule Correctors and Transcomplementation. PLoS One 10, e0119796.

Riordan JR. (2008). CFTR Function and Prospects for Therapy. Annu Rev Biochem 77, 701-726.

Rowe SM, Pyle LC, Jurkevante A, Varga K, Collawn J, Sloane PA, et al. (2010). DeltaF508 CFTR Processing Correction and Activity in Polarized Airway and Non-Airway Cell Monolayers. Pulm Pharmacol Ther 23, 268-278.

Roxo-Rosa M, Xu Z, Schmidt A, Neto M, Cai Z, Soares CM, et al.(2006). Revertant Mutants G550E and 4RK Rescue Cystic Fibrosis Mutants in the First Nucleotide-Binding Domain of CFTR by Different Mechanisms. Proc Natl Acad Sci U S A 103, 17891-17896.

Sampson HM, Robert R, Liao J, Matthes E, Carlile GW, Hanrahan JW, et al. (2011). Identification of a NBD1-binding Pharmacological Chaperon that Corrects the Trafficking Defect of F508del-CFTR. Chem Biol 18, 231-242.

Serohijos AWR, Hegedus T, Aleksandrov AA, He L, Cui L, Dokholyan NV,et al. (2008). Phenylalanine-508 Mediates a Cytoplasmic-Membrane Domain Contact in the CFTR 3D Structure Crucial to Assembly and Channel Function. Proc Natl Acade Sci U S A 105, 3256-3261.

Sheppard DN, Rich DP, Ostedgaard LS, Gregory RJ, Smith AR, Welsh MJ. (1993). Mutations in CFTR associated with mild-disease-form Cl- channels with altered pore properties. Nature 362, 160-164. 
Sondo E, Tomati V, Caci E, Esposito AI, Pfeffer U, Pedemonte N, et al. (2011). Rescue of the Mutant CFTR Chloride Channel by Pharmacological Correctors and Low Temperature Analyzed by Gene Expression Profiling. Am J Physiol Cell Physiol 301, C872-C885.

Taylor-Cousar JL, Munck A, McKone EF, van der Ent CK, Moeller A, Simard C, et al. (2017). TezacaftorIvacaftor in Patients with Cystic Fibrosis Homozygous for Phe508del. N Eng J Med 377, 2013-2023.

Thibodeau PH, Richardson JM $3^{\text {rd }}$, Wang W, Millen L, Watson J, Mendonza JL, et al. (2010). The cystic fibrosis-causing mutation delF508 affects multiple steps in cystic fibrosis transmembrane conductance regulator biogenesis. J Biol Chem 285, 35825-25835.

Turner MJ, Luo Y, Thomas DY, Hanrahan JW. (2020). The Dual Phosphodiesterase $\frac{3}{4}$ Inhibitor RPL554 Stimulates Rare Class III and IV CFTR Mutants. Am J Physiol Lung Cell Mol Physiol, doi: 10.1152/ajplung.00285.2019.

Veit G, Avramescu RG, Perdomo D, Phuan PW, Bagdany M, Apaja PM, et al. (2014). Some gating potentiators, including VX-770, diminish $\Delta$ F508-CFTR functional expression. Sci Transl Med 6, 246ra97.

Wainwright CE, Elborn JS, Ramsey BW, Marigowda G, Huang X, Cipolli M, et al. (2015). LumacaftorIvacaftor in Patients with Cystic Fibrosis Homozygous for Phe508del CFTR. N Engl J Med 373, 220-231.

Wang X, Matteson J, An Y, Moyer B, Yoo JS, Bannykh S, et al.(2004). COPII-dependent export of cystic fibrosis transmembrane conductance regulator from the ER uses a di-acidic exit code. J Cell Biol 167,65-74.

Figure legends:

Figure 1 - RDR01752 rescues F508del-CFTR processing and PM expression. (A) CFBE cells stably expressing F508del-CFTR were incubated for $24 \mathrm{~h}$ with DMSO (negative control), VX-809 (3.7 $\mu \mathrm{M})$, VX-661 $(5 \mu \mathrm{M})$ or an increasing concentration of RDR01752. (B) CFTR processing (C/B+C) was quantified and normalized to tubulin levels (loading control). $\mathrm{N}=4$. Vs. DMSO: ${ }^{*} P<0.05,{ }^{* * *} P<0.001$. Vs. VX809: $P<<0.05$, $\# \#<<0.01$. (C) CFBE stably expressing mCherry-Flag-F508del-CFTR were incubated for $48 \mathrm{~h}$ with DMSO (negative control), VX-809 $(3.7 \mu \mathrm{M})$ or an increasing concentration of RDR01752. (D) Immunostaining was performed and fluorescence images of extracellularly exposed Flag-tags were quantified to determine CFTR PM expression. Data are normalized to the negative control (DMSO). $\mathrm{N}=4 . \quad V s$. VX-809: ${ }^{\#} P<0.05,{ }^{\# \#} P<0.01$.

Figure 2 - RDR01752 rescues F508del-CFTR function. (A)Representative cell fluorescence recording acquired with a microplate reader. CFBE cells stably co-expressing F508del-CFTR and the HS-YFP were incubated for $24 \mathrm{~h}$ with DMSO (vehicle), VX-809 $(3.7 \mu \mathrm{M})$ or increasing concentrations of RDR01752. Cells were then acutely $(30 \mathrm{~min})$ stimulated with Fsk $(20 \mu \mathrm{M})$ and Gen $(50 \mu \mathrm{M})$. (B) CFTR activity was quantified based on the rate of YFP quenching and normalized to the negative control (DMSO, dashed line). $\mathrm{N}=4$. Vs. VX-809:\# $P<0.05$. (C-F) Monolayers of CFBE cells stably expressing F508del-CFTR were incubated for $24 \mathrm{~h}$ with (C) DMSO (negative control), (D) VX-809 (3.7 $\mu \mathrm{M})$, or (E) RDR01752 $(10 \mu \mathrm{M})$. Original Ussing chamber (open-circuit) recordings depicting transepithelial voltage measurements $\left(\mathrm{V}_{\mathrm{te}}\right)$. There is an absence of response in cells treated with DMSO, while negative deflections are observed in cells treated with VX-809 or RDR01752 following the application of Fsk+IBMX and genistein, which are reverted by application of $\operatorname{Inh}_{172} .(\mathbf{F})$ Data are expressed as Isc calculated from voltage deflections obtained for the responses to Fsk+IBMX+Gen. $\mathrm{N}=3$. Vs. DMSO: ${ }^{*} P<0.05,{ }^{*} P<0.01$. Vs. VX-809: ${ }^{*} P<0.05$.

Figure 3 - Intestinal organoids (F508del/F508del) respond positively to RDR01752. (A) Brightfield images of organoids incubated for $24 \mathrm{~h}$ with DMSO (negative control), VX-809 (3.7 $\mu \mathrm{M})$, VX-661 (5 $\mu \mathrm{M})$ or RDR01752 $(10 \mu \mathrm{M})$ and acutely stimulated with forskolin (Fsk, $0.128 \mu \mathrm{M})$ with VX-770 $(3 \mu \mathrm{M})$ or genistein $(\mathrm{Gen}, 50 \mu \mathrm{M})$. (B) Data of FIS of organoids are expressed as the absolute area under the curve (AUC; baseline $=100 \%, t=60 \mathrm{~min}, 0.128 \mu \mathrm{M}$ Fsk). Absence of bars indicates there was no swelling (NS). $\mathrm{N}=3$. Vs. DMSO: ${ }^{*} P<0.05,{ }^{* *} P<0.01,{ }^{* * *} P<0.001$. 
Figure 4-RDR01752 increases the rescue of F508del-CFTR PM expression in low temperature and in cells expressing in cis the genetic revertants G550E and 4RK, but not in R1070W. CFBE stably expressing mCherry-Flag-CFTR (WT, F508del, DD/AA variants or carrying G550E, R1070W, 4RK in cis with F508del) were incubated for $24 \mathrm{~h}$ with DMSO (negative control), RDR01752 (10 $\mu \mathrm{M})$, VX-809 $(3.7 \mu \mathrm{M})$ or VX-661 $(5 \mu \mathrm{M})$ and then maintained for additional $24 \mathrm{~h}$ at $(\mathbf{A}) 37^{\circ} \mathrm{C}$ or $(\mathbf{B})$ in low temperature $\left(27^{\circ} \mathrm{C}\right)$. Data are represented as heatmaps of the quantification from fluorescence images of extracellularly exposed Flag-tags to determine CFTR PM expression. The values for each cell line are normalized to DMSO at $37^{\circ} \mathrm{C} . \mathrm{N}=6$.

Figure 5 - RDR01752 is not additive to VX-809 or VX-661 in rescuing F508del-CFTR traffic. (A) CFBE stably expressing mCherry-Flag-F508del-CFTR were incubated for $48 \mathrm{~h}$ with the following compounds individually or in combination: DMSO (negative control), RDR01752 (10 $\mu \mathrm{M})$, VX-809 (3.7 $\mu \mathrm{M})$, VX-661 $(5 \mu \mathrm{M})$ and VX-770 $(3 \mu \mathrm{M})$.(B) Immunostaining was performed and fluorescence images of extracellularly exposed Flag-tags were quantified to determine CFTR PM expression. Data are normalized to the negative control (DMSO). $\mathrm{N}=4$. Vs. single corrector (white bars): ${ }^{*} P<0.05$, ${ }^{* * *} P<0.001$. n.s.: no significant.

Figure 6 - Assessment of RDR01752 effects on rare CFTR mutants by FLIPR membrane potential (FMP) assay. FRT cells stably expressing CFTR variants (wt, G85E, R334W, T338I, R347P, F508del, V520F, S549F, G551D, M1101K or N1303K) were incubated for $24 \mathrm{~h}$ with DMSO (negative control), RDR01752 $(10 \mu \mathrm{M})$ or VX-809 $(1 \mu \mathrm{M})$. FMP assay was performed to monitor membrane depolarization induced by stimulation with forskolin $(10 \mu \mathrm{M})$ plus genistein $(50 \mu \mathrm{M})$ as a measurement of CFTR function. $\mathrm{N}=3 . V s$. DMSO (black bars): ${ }^{*} P<0.05$.

Figure 7 - Effect of RDR01752 and VX-809 individually or in combination on functional rescue of CFTR carrying F508del, G85E, N1303K or R334W. (A) Monolayers of FRT cells stably expressing CFTR variants (F508del, G85E, N1303K or R334W) were incubated for $24 \mathrm{~h}$ with DMSO (negative control), VX-809 $(1 \mu \mathrm{M})$ and RDR01752 $(10 \mu \mathrm{M})$ alone or combined. Representative recordings of Isc measurements of Ussing chamber for each CFTR mutant. CFTR currents were stimulated using forskolin (FSK; $10 \mu \mathrm{M})$ and genistein $(\mathrm{GST} ; 50 \mu \mathrm{M})$ and inhibited by CFTR $_{\text {inh }-172}(172 ; 10 \mu \mathrm{M})$. ATP $(100 \mu \mathrm{M})$ was added at the end of each experiment as a positive control for viability. (B)Data are represented as mean increase in Isc induced by FSK+GST. $\mathrm{N}=3-6$. Vs. DMSO: ${ }^{*} P<0.05$, ${ }^{* *} P<0.01$.

\section{Supplementary Figures:}

Supplementary Figure 1 - Intestinal organoids (F508del/F508del) respond positively to RDR01752. Organoids were incubated for $24 \mathrm{~h}$ with DMSO (negative control), VX-809 (3.7 $\mu \mathrm{M})$, VX-661 (5 $\mu \mathrm{M})$ or RDR01752 $(10 \mu \mathrm{M})$ and acutely stimulated with different concentrations of forskolin (Fsk, 0.128 to $20 \mu \mathrm{M}$ ) with VX-770 $(3 \mu \mathrm{M})$ or genistein $(\mathrm{Gen}, 50 \mu \mathrm{M})$. Graph summarizing data of FIS expressed as the absolute area under the curve (AUC; baseline $=100 \%, t=60 \mathrm{~min}$ ). $\mathrm{N}=3$.

Supplementary Figure 2 - RDR01752 is not additive to C18 in rescuing F508del-CFTR traffic. (A) CFBE stably expressing mCherry-Flag-F508del-CFTR were incubated for $48 \mathrm{~h}$ with the following compounds individually or in combination: DMSO (negative control), RDR01752 (10 $\mu \mathrm{M}), \mathrm{C} 18(5 \mu \mathrm{M})$ and VX-770 $(3 \mu \mathrm{M})$. (B)Immunostaining was performed and fluorescence images of extracellularly exposed Flag-tags were quantified to determine CFTR PM expression compared to the DMSO. $\mathrm{N}=4$. Vs. single corrector (white bars): ${ }^{*} P<0.05,{ }^{* * *} P<0.001$. n.s.: not significant. 
A

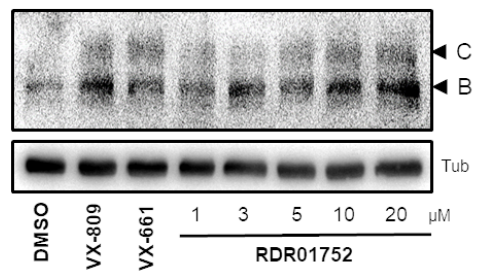

B

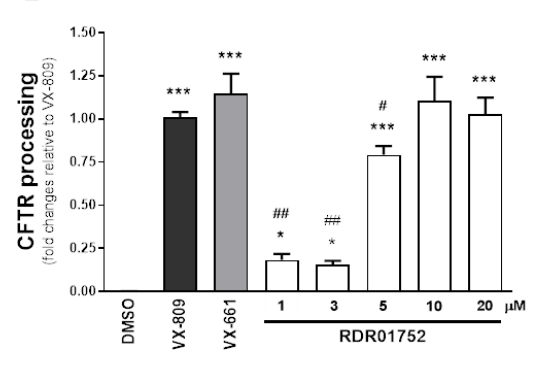

C

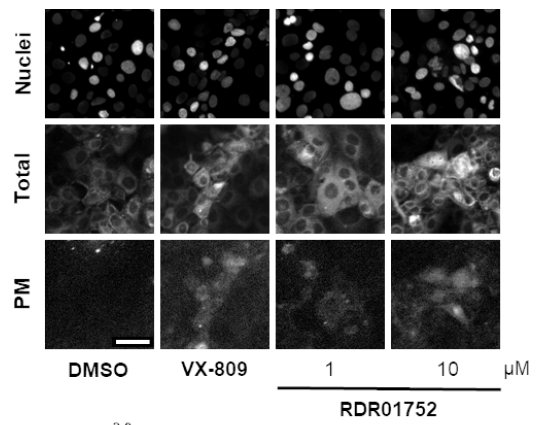

D

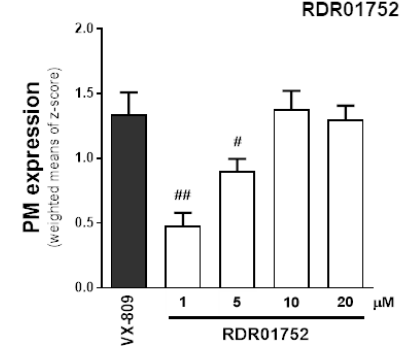



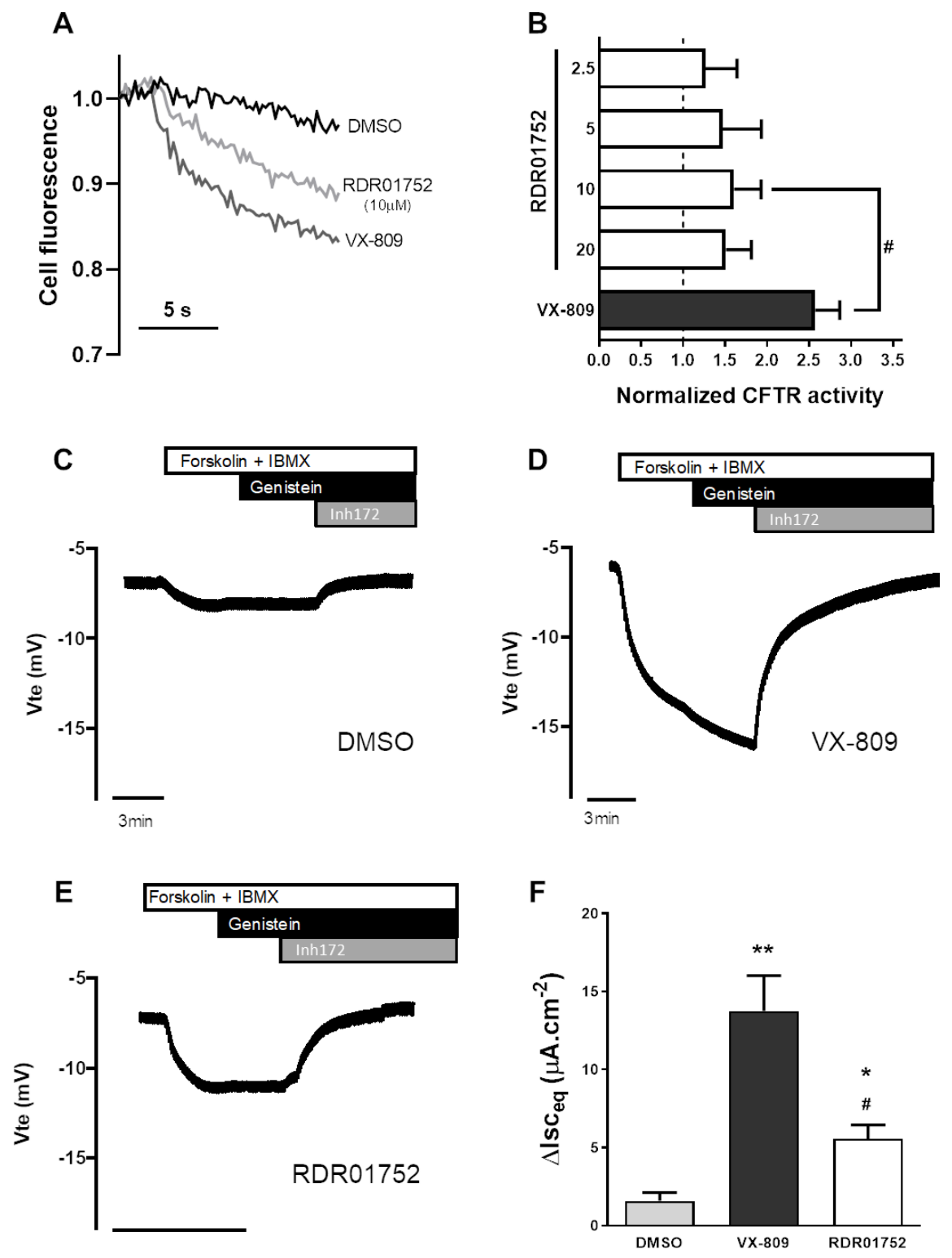

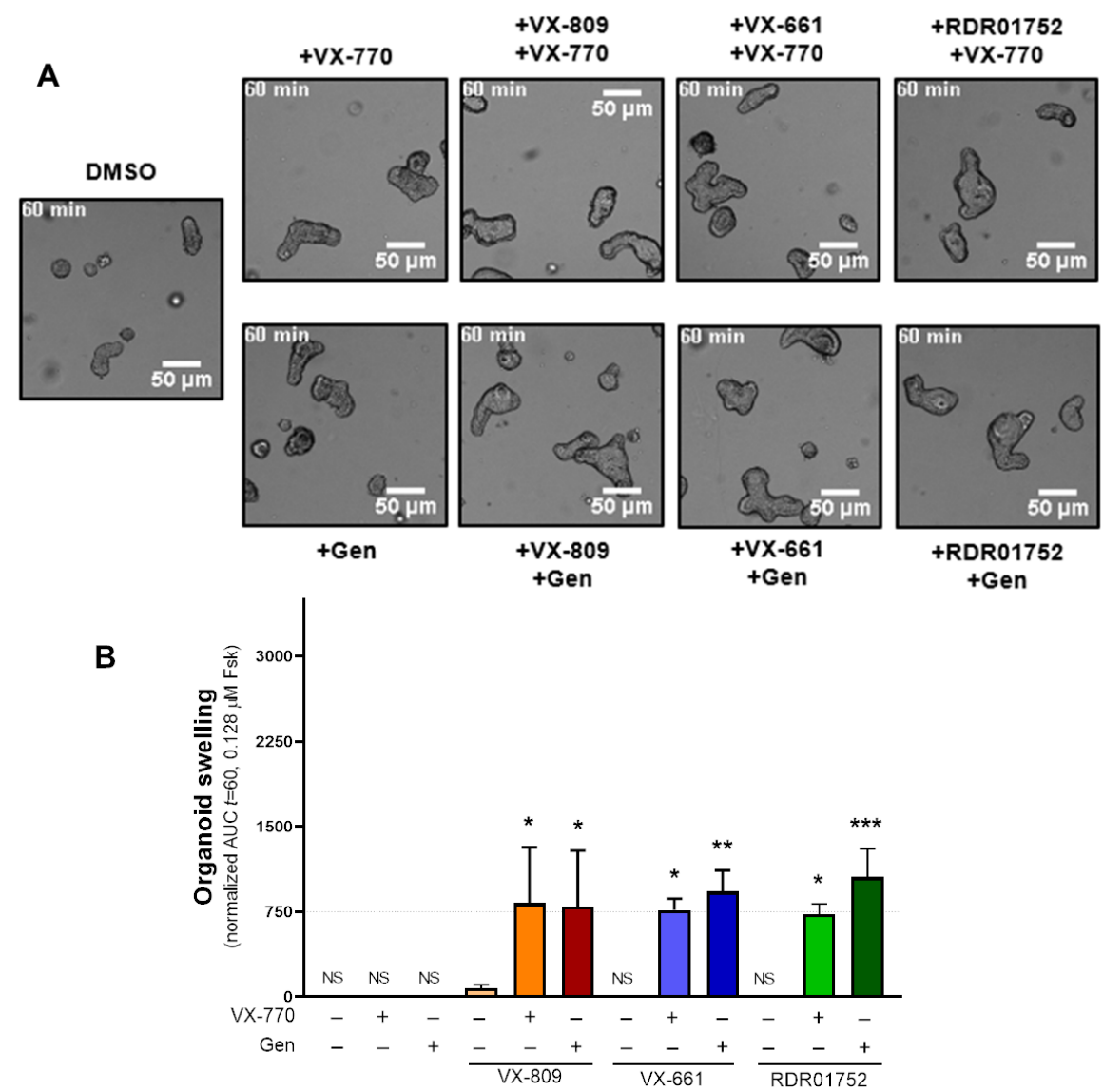

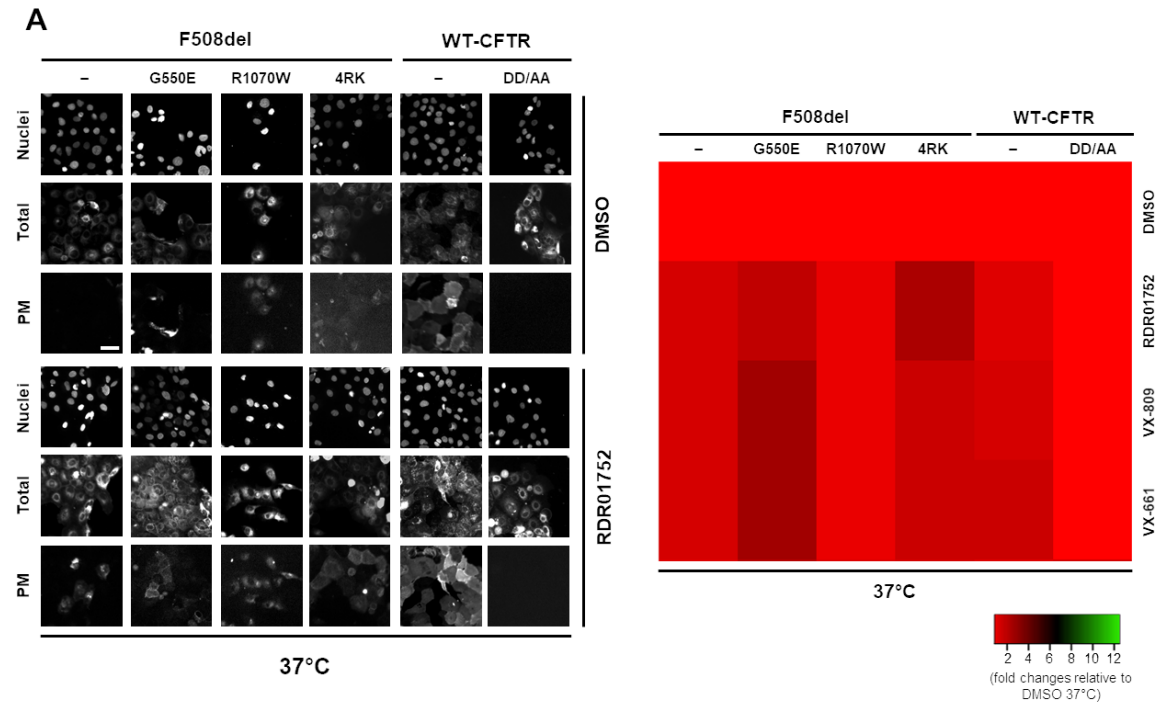

B $\frac{\text { WT-CFTR }}{-\quad \text { DD/AA }}$
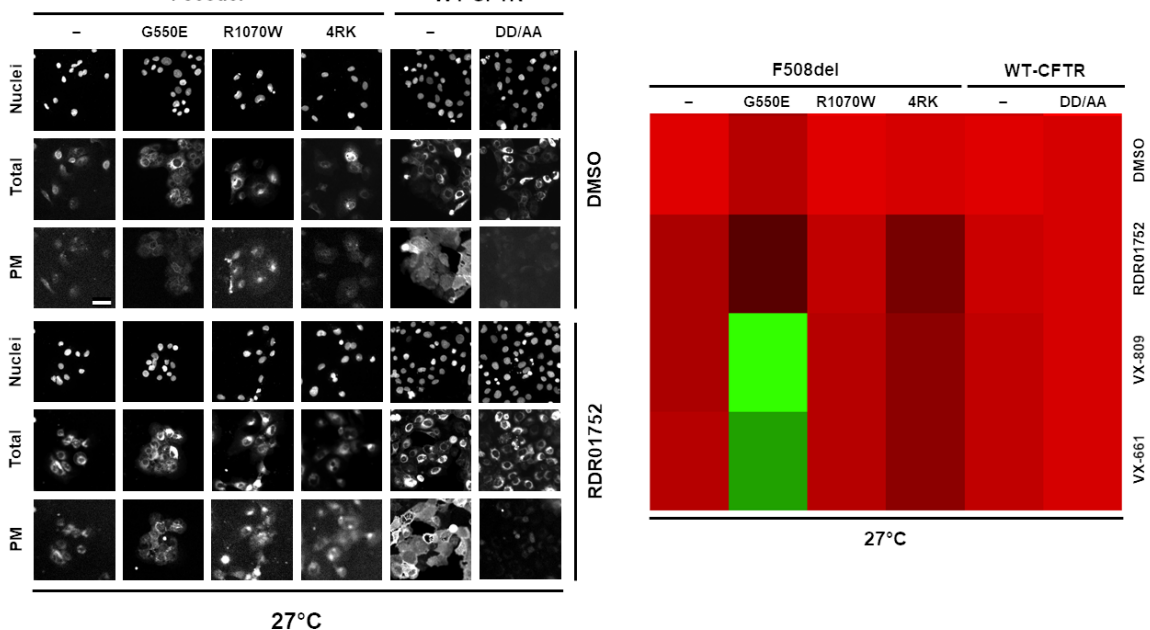

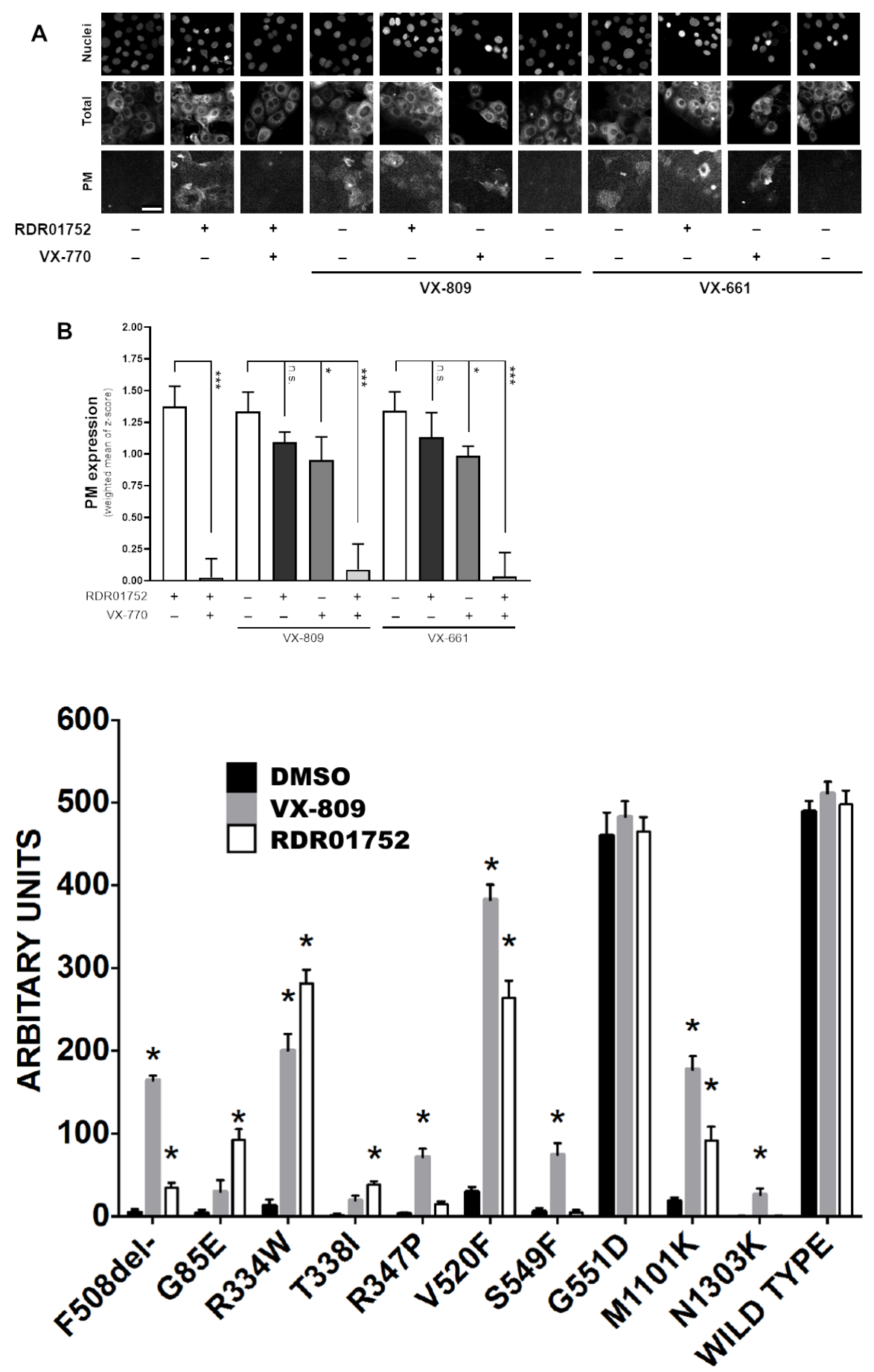


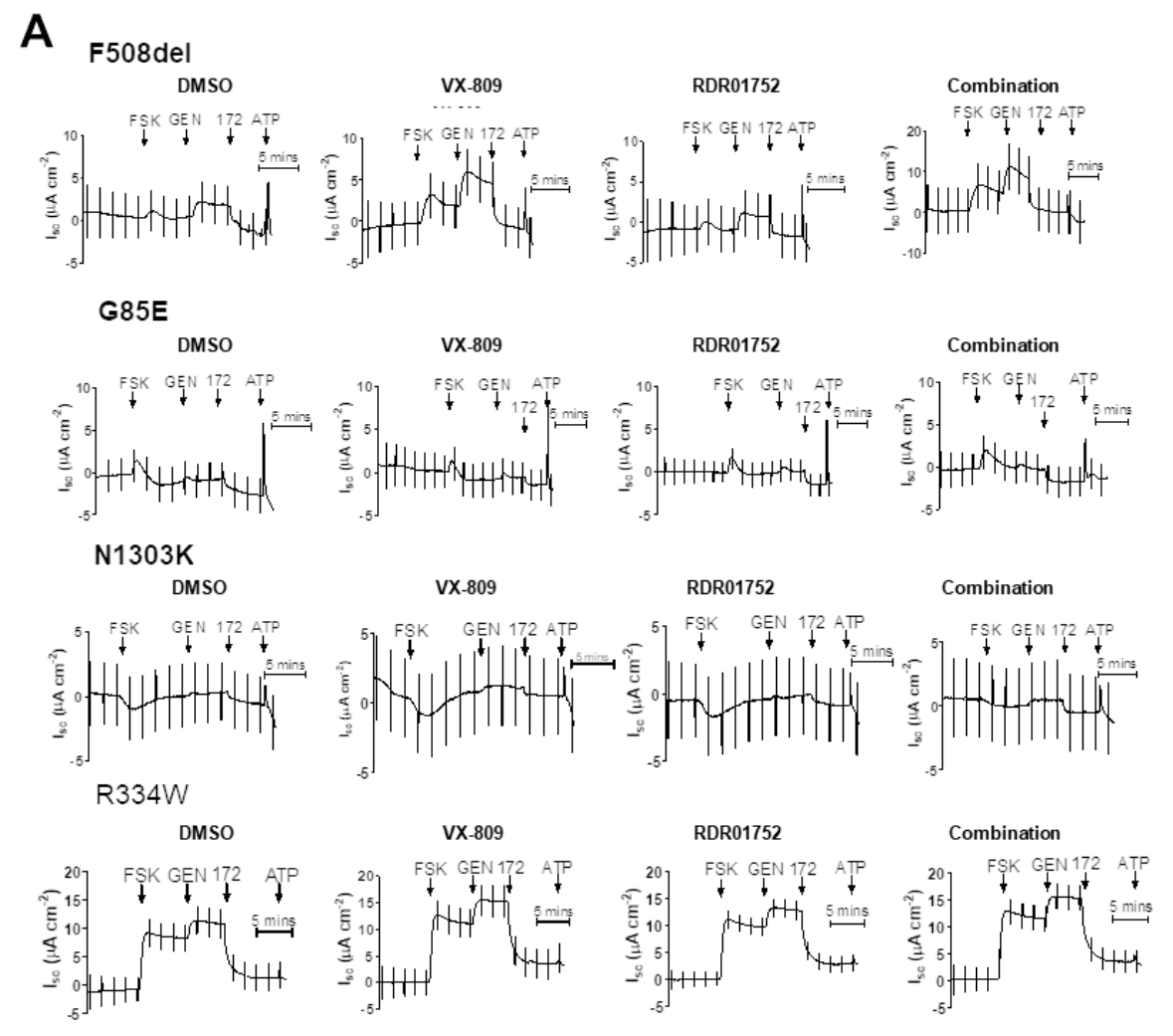

B

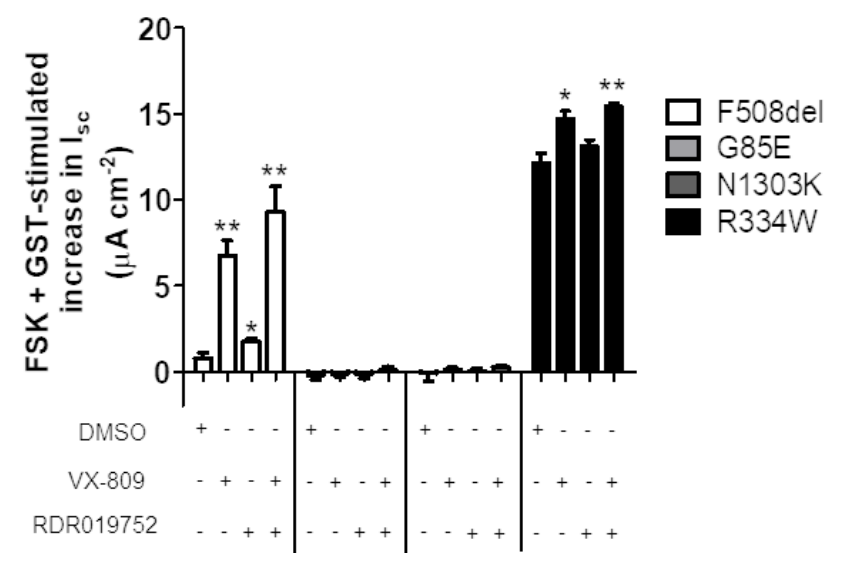

